\title{
Genetic Diversity Studies in Finger Millet under Terminal Moisture Stress
}

\author{
V.T. Manjunatha ${ }^{1}$, L.N. Yogeesh ${ }^{2 *}$, J.P. Nidagundi ${ }^{1}$, G. Girish ${ }^{2}$ and T.C. Suma ${ }^{3}$ \\ ${ }^{1}$ Department of Genetics and Plant Breeding, UAS, Raichur, India \\ ${ }^{2}$ Agricultural Research Station, UAS, Raichur, Hagari, Ballari, India \\ ${ }^{3}$ Department of Crop Physiology, UAS, Raichur, Karnataka, India \\ *Corresponding author
}

\section{Keywords \\ Moisture stress free condition (MSF), \\ Terminal moisture stress (TMS) \\ Article Info \\ Accepted: \\ 10 November 2020 \\ Available Online: \\ 10 December 2020}

\section{A B S T R A C T}

The present investigation is carried out to study the genetic divergence among 108 Ragi genotypes for fifteen different characters using Mahalanobis $\mathrm{D}^{2}$ statistics. The 108 finger millet genotypes were grouped into 7 clusters and 8 clusters under moisture stress free (MSF) and terminal moisture stress (TMS) conditions respectively, irrespective of geographical diversity, indicating no parallelism between geographic and genetic diversity. Under terminal moisture stress (TMS) condition, highest number of genotypes were observed in cluster I (33) followed by cluster III (28), cluster II (21), cluster IV (17), cluster V (8), cluster VIII (2) and remaining clusters such as clusters VI, cluster VII were solitary clusters and the highest inter-cluster distance was observed between cluster III and cluster VIII indicating wider range of variability and the genotypes of respective clusters can be utilized for introgressing useful traits for the development of elite cultivars.

\section{Introduction}

Finger millet (Eleusine coracana L.) is a crop of antiquity and known for their suitability to dry lands and tribal agriculture of sustainable nature. The resilience exhibited by this crop is helpful in their adjustment to different ecological situations making it an ideal crop for climate change and contingency
planning.Finger millet belongs to family Poaceae, subfamily Chloridoideae and genus Eleusine. It is native to East Africa and came to India around $2000 \mathrm{BC}$. It is widely grown in arid and semiarid areas of Africa and Asia. In India, it ranks sixth in production after wheat, rice, maize, sorghum and bajra. India is a major producer of Ragi in Asia with a cultivated area of 1.19 million hectares, 
production of 1.98 million tonnes giving an average productivity of about $1661 \mathrm{~kg}$ per hectare. The major finger millet growing states are Karnataka, Maharashtra, Uttarakhand, Tamil Nadu, Simandra, Orissa and Jharkhand. It is mainly grown and consumed in Karnataka, Andhra Pradesh, Tamil Nadu, Odisha, Maharashtra, Uttarakhand and Goa. In Karnataka, it is popularly known as "Ragi" grown in an area of 0.598 million hectares with a production of 0.86 million tones. Finger millet is considered to be one of the most nutritious cereals which contains about $65-75 \%$ carbohydrates, $5-8 \%$ protein, 15-20\% dietary fibers and $2.5-3.5 \%$ minerals, $1-2 \%$ ether extractives. Of all the cereals and millets, finger millet has the highest amount of calcium (344 mg) and potassium (408 mg) (Devi et al., 2014). Finger millet can be termed as smart food being "good for you, the planet and the farmer" and are fast becoming a favourite globally. With its high nutrient profile and being versatile in nature it is also known to be a promising immunity booster amid COVID19 pandemic.

With the climate change looming large, shifts in rainfall pattern and vagaries of monsoon the crop frequently confronts drought stress (DS) at flowering and grain filling stages of the crop cycle is referred to "Terminal drought". Terminal drought stress is the consequence of the crop growing and maturing in a progressively depleting soil moisture profile (Ludlow and Muchow, 1990; Krishnamurthy et al., 1999). Acute water shortage is a threat to crop cycle and when this condition often coincides under arid and semiarid climates it causes a severe threat to productivity by limiting the rate and duration of grain filling growth. The present study was undertaken during Rabi 2019 at Agricultural Research Station, Hagari, Ballari, Karnataka to know genetic diversity and to identify superior genotypes for yield and its component traits under Terminal Moisture Stress (TMS) among finger millet accessions.

\section{Materials and Methods}

The experimental material consisted of one hundred eight Ragi genotypes, along with three checks viz: ML365, GPU67 and GPU28. Two experiments were under taken in the augmented design where the Finger millet genotypes were raised in Moisture Stress Free (MSF) \& Terminal Moisture Stress (TMS) environmental conditions. Each genotype was grown in $2 \mathrm{~m}$ long single row plot. Observations were recorded for fifteen characters viz., days to fifty per cent flowering, days to maturity, plant height $(\mathrm{cm})$, productive tillers per plant, finger length $(\mathrm{cm})$, fingers per ear head, 1000 seed weight $(\mathrm{g})$, harvest index $(\%)$, root length $(\mathrm{cm})$, root volume (cc), root biomass $(\mathrm{g})$, proline ( $\mu$ mole $\mathrm{g}^{-1}$ fr.wt), RWC (\%), SCMR and grain yield per plant $(\mathrm{g})$ on five competitive plants selected at random for each genotypes. The mean values on these observations were subjected for statistical analysis and the genetic divergence was computed using Mahalanobis $\mathrm{D}^{2}$ statistics. Based on genetic distance, all the genotypes were grouped into different clusters (Rao, 1952).

\section{Results and Discussion}

One hundred eight genotypes of finger millet were grouped into 7 clusters and 8 clusters under moisture stress free (MSF) and terminal moisture stress (TMS) conditions respectively (Table 1) and (Table 2). Under moisture stress free (MSF) condition, cluster I (56) recorded highest number of genotypes followed by cluster II (28) and cluster IV (23) while cluster III, V, VI, and VII were solitary clusters. Under terminal moisture stress (TMS) condition, highest number of genotypes was observed in cluster I (33) followed by cluster III (28), cluster II (21), 
cluster IV (17), cluster V (8), cluster VIII (2) and remaining clusters such as clusters VI, cluster VII were solitary clusters. Genotypes of different geographical areas fall in one group and also the genotypes of same geographical area were clubbed into different groups indicating there is no formed relationship between geographical diversity and genetic diversity (Surnarayana et al., 2014; Devaliya et al., 2017 and Patil et al., 2018).

Intra and inter cluster $\mathrm{D}^{2}$ values were worked out using $\mathrm{D}^{2}$ values from divergence analysis for both moisture stress free (MSF) (Table 3) and terminal moisture stress (TMS) conditions (Table 4). Under moisture stress free (MSF), cluster IV (741.23) had maximum intra cluster distance followed by cluster I (577.91) and cluster II (510.14). This implies that these clusters have the genotypes with varied genetic architecture. Cluster III, cluster V, cluster VI and cluster VII showed zero intra cluster distance due to monogenotypic nature. High intra-cluster genetic distance in cluster IV was because of heterogeneous composition of that cluster. Likely under terminal moisture stress (TMS) condition, cluster V (1278.03) had maximum intra cluster distance followed by cluster IV (880.011), cluster III (780.91), cluster II (707.79), cluster VIII (608.14) and cluster I (536.98). While, cluster VI and cluster VII showed zero intra cluster distance due to monogenotypic nature under moisture stress.

Under moisture stress free (MSF) environment, maximum inter-cluster distance was observed between cluster IV and cluster VII (3763.54) followed by cluster III and cluster VI (3285.3), cluster II and IV (3130.41), cluster II and cluster V (2703.06), cluster IV and cluster VI (2408.84), cluster III and cluster VI (2231) and cluster II and cluster III (2043.47). The inter cluster between cluster II and cluster VI (1936.02), cluster V and cluster VII (1826.24), cluster III and cluster V (1708.64), cluster I and cluster VII (1516.1), cluster I and cluster II (1465.51), cluster IV and cluster V (1358.64), cluster I and cluster IV (1260.48), cluster II and cluster VII (1148.24), cluster VI and cluster VII (1010.98) and cluster I and cluster VI (1010.14) were also high. The clusters with higher inter-cluster distances indicated that the genotypes included in those clusters had high genetic variation and hybridization between genotypes of these clusters may result heterotic hybrids because of convergence of diverse genes scattered in parents to progeny (Rawat et al., 2018). The minimum estimate for inter-cluster distance were recorded between cluster $\mathrm{V}$ and cluster VI (617.16) followed by cluster I and cluster V (821.14), cluster III and cluster IV (873.57) and cluster I and cluster III (903.78).

Under terminal moisture stress (TMS) condition, maximum inter-cluster distance was observed between cluster III and cluster VIII (7828.66) followed by cluster $\mathrm{V}$ and cluster VIII (5718.33), cluster VII and VIII (5177.83), cluster VI and cluster VIII (4953.03), cluster I and cluster VIII (4306.52), cluster III and cluster IV (3480.8), cluster II and cluster VIII (2826.01), cluster IV and cluster V (2808.3), cluster IV and cluster VII (2781.26), cluster II and cluster III (2419.4), cluster IV and cluster VI (2373.62), cluster V and cluster VII (2210.03) and cluster III and cluster V (2162.11). The inter cluster between cluster IV and cluster VIII (1938.55), cluster V and cluster VI (1907.19), cluster I and cluster VII (1823.34), cluster II and cluster V (1706.39), cluster III and cluster VII (1644.16), cluster I and cluster III (1551.25), cluster I and cluster IV (1502.82), cluster I and cluster VI (1423.49), cluster II and cluster VII (1373.49), cluster III and cluster VI (1252.06) were also high. The minimum estimate for inter-cluster distance were recorded between cluster II and cluster 
IV (1195.42), cluster I and cluster V (1162.53), cluster II and cluster VI (1137.28), cluster I and cluster II (1023.44) and cluster VI and cluster VII (227.75). The clusters with lowest inter- cluster distances indicated that genotypes present in these cluster pairs were genetically close to each other. The crosses between genotypes belonging to clusters separated by low inter cluster distance were likely to throw promising recombinants in the segregating generations. Wolie et al., (2013) suggested that most diverse cluster may be used as parents in hybridization programme to develop high yielding varieties, while selection and choice of parents mainly depends upon contribution of characters towards divergence as stated by Dinesh et al., (2010).

Cluster means for all fifteen characters under moisture stress free (MSF) and terminal moisture stress (TMS) conditions are depicted in Table 5 and Table 6 respectively. The results indicated appreciable difference among clusters means for most of the characters.

Under moisture stress free (MSF), as far as cluster means are concerned, cluster II had the lowest mean values for days to fifty per cent flowering, days to maturity and 1000 seed weight. Cluster III had the highest mean values root length, root volume and root biomass, while it showed lowest mean values for characters finger length, harvest index, proline, RWC, SCMR and grain yield. Cluster IV had the highest mean value for plant height. Cluster V had the highest mean values for days to fifty per cent flowering, proline and RWC. Cluster VI had the highest mean values for days to maturity, productive tillers per plant, fingers per ear head and SCMR. Cluster VII had the highest mean values for finger length, harvest index and grain yield.

Table.1 Clustering pattern of finger millet genotypes under moisture stress free environment

\begin{tabular}{|c|c|c|}
\hline Clusters & $\begin{array}{c}\text { No. of } \\
\text { genotypes }\end{array}$ & Genotypes \\
\hline $\mathbf{I}$ & 56 & $\begin{array}{l}\text { HR55,HR53,HR51,HR39,HR32,HR23,HR31,HR38,HR14,HR60,HR59 } \\
\text { HR58,HR2,HR34,HR33,HR12,HR20,HR3,HR9,HR40,HR16,HR11,HR5 } \\
\text { HR21,HR57,HR6,KMR652,PR1639,ML365,HR24,HR41,HR42,WN562 } \\
\text { HR19,HR56,TNEC1297,TNEC1299,HR18,HR54,TNEC1302,HR30,HR37 } \\
\text { KMR650,GPU28,HR35,HR28,HR22,HR61,HR50,GPU99,HR36,HR17 } \\
\text { HR4,HR26,GPU98,WN591. }\end{array}$ \\
\hline II & 28 & $\begin{array}{l}\text { PPR1082,PR1506,VR1125,VL376,VR1112,VL400,VL399, BR14-2 } \\
\text { PRSW43,GPU101,RAUF21,OEB608,KOPN1112, IIMR-R18-5725 } \\
\text { PRS38,TNEC1311,DPLN2,KWFM47, PPR1091, PR202, IIMRFM8023, } \\
\text { GPU100,IIMRFM8011, KMR704, KMR703,HR13,BR14-27,GSMC1 }\end{array}$ \\
\hline III & 1 & GPU 45 \\
\hline IV & 23 & $\begin{array}{l}\text { HR27,HR49,HR46,HR1,VR1110,HR45,HR15,HR25, RAUF17, } \\
\text { OEB604, HR48,GPU67,VL382,HR10,VR117, VL394, } \\
\text { VR1125, HR29, PR202, HR44, HR7, HR43, HR19 }\end{array}$ \\
\hline $\mathbf{V}$ & 1 & HR 8 \\
\hline VI & 1 & HR 13 \\
\hline VII & 1 & HR 52 \\
\hline
\end{tabular}


Table.2 Clustering pattern of finger millet genotypes under terminal moisture stress environment

\begin{tabular}{|c|c|l|}
\hline Clusters & $\begin{array}{c}\text { No. of } \\
\text { genotypes }\end{array}$ & \multicolumn{1}{|c|}{ Genotypes } \\
\hline $\mathbf{I}$ & 33 & $\begin{array}{l}\text { HR39,HR20,HR23,HR38,HR14,HR32,HR31,HR55,HR19, HR53, } \\
\text { HR50,HR58,HR59,HR56,HR2,HR40,HR9,HR3,HR46HR42,HR51, } \\
\text { HR12,HR34,HR37,HR35,HR60,ML365,RAUF17,HR6,HR61,HR41, } \\
\text { HR30,HR22 }\end{array}$ \\
\hline II & 21 & $\begin{array}{l}\text { HR33,HR57,HR36,HR11,VR117,GPU45,GPU98,HR43, HR16, } \\
\text { KMR652,GPU99,KMR650,PR1639,GPU67,GPU28, GSMC1, } \\
\text { WN591,HR24,VR1110,PR202,OEB604 }\end{array}$ \\
\hline III & 28 & $\begin{array}{l}\text { PRSW43,RAUF21,OEB608,VL399,VL376,KOPN1112, GPU100, } \\
\text { VL400,BR142,VR1125,VR1112,PR1506,PR202, KWFM47, } \\
\text { GPU101,TNEC1297,TNEC1311,IIMR R18-5725, PPR1082, } \\
\text { TNEC1299,IIMRFM823,TNEC1302,KMR703,PRS38,IMRFM811, } \\
\text { PPR1091,DPLN2,HR13 }\end{array}$ \\
\hline IV & 17 & $\begin{array}{l}\text { HR25,HR26,VL382,HR10,HR44,HR27,HR1,WN562,HR45,HR15, } \\
\text { HR49, HR29,VL376,HR7,VL394,HR5,HR8 }\end{array}$ \\
\hline $\mathbf{V}$ & 8 & HR21,HR54,HR18,HR28,HR13,HR4,HR47,HR52 \\
\hline VI & 1 & BR14-27 \\
\hline VII & 1 & KMR704 \\
\hline VIII & 2 & HR43,HR48 \\
\hline
\end{tabular}

Table.3 Intra (diagonal) and inter-cluster $\mathrm{D}^{2}$ values among seven clusters in finger millet under moisture stress free environment

\begin{tabular}{|c|c|c|c|c|c|c|c|}
\hline Clusters & I & II & III & IV & V & VI & VII \\
\hline I & $\mathbf{5 7 7 . 9 1}$ & 1465.51 & 903.78 & 1260.48 & 821.14 & 1010.14 & $\mathbf{1 5 1 6 . 1}$ \\
\hline II & 1465.51 & $\mathbf{5 1 0 . 1 4}$ & 2043.47 & 3130.41 & 2703.06 & 1936.02 & $\mathbf{1 1 4 8 . 2 4}$ \\
\hline III & 903.78 & 2043.47 & $\mathbf{0}$ & 873.57 & 1708.64 & 2231 & $\mathbf{3 2 8 5 . 3}$ \\
\hline IV & 1260.48 & 3130.41 & 873.57 & $\mathbf{7 4 1 . 2 3}$ & 1358.64 & 2408.84 & $\mathbf{3 7 6 3 . 5 4}$ \\
\hline V & 821.14 & 2703.06 & 1708.64 & 1358.64 & $\mathbf{0}$ & 617.16 & $\mathbf{1 8 2 6 . 2 4}$ \\
\hline VI & 1010.14 & 1936.02 & 2231 & 2408.84 & 617.16 & $\mathbf{0}$ & $\mathbf{1 0 1 0 . 9 8}$ \\
\hline VII & $\mathbf{1 5 1 6 . 1}$ & $\mathbf{1 1 4 8 . 2 4}$ & $\mathbf{3 2 8 5 . 3}$ & $\mathbf{3 7 6 3 . 5 4}$ & $\mathbf{1 8 2 6 . 2 4}$ & $\mathbf{1 0 1 0 . 9 8}$ & $\mathbf{0}$ \\
\hline
\end{tabular}


Table.4 Intra (diagonal) and inter-cluster $\mathrm{D}^{2}$ values among eight clusters in finger millet under terminal moisture stress environment

\begin{tabular}{|c|c|c|c|c|c|c|c|c|}
\hline Clusters & I & II & III & IV & V & VI & VII & VIII \\
\hline I & $\mathbf{5 3 6 . 9 8}$ & 1023.44 & 1551.25 & 1502.82 & 1162.53 & 1423.49 & 1823.34 & $\mathbf{4 3 0 6 . 5 2}$ \\
\hline II & 1023.44 & $\mathbf{7 0 7 . 7 9}$ & 2419.4 & 1195.42 & 1706.39 & 1137.28 & 1373.49 & $\mathbf{2 8 2 6 . 0 1}$ \\
\hline III & 1551.25 & 2419.4 & $\mathbf{7 8 0 . 9 1}$ & 3480.8 & 2162.11 & 1252.06 & 1644.16 & $\mathbf{7 8 2 8 . 6 6}$ \\
\hline IV & 1502.82 & 1195.42 & 3480.8 & $\mathbf{8 8 0 . 0 1}$ & 2808.3 & 2373.62 & 2781.26 & $\mathbf{1 9 3 8 . 5 5}$ \\
\hline V & 1162.53 & 1706.39 & 2162.11 & 2808.3 & $\mathbf{1 2 7 8 . 0 3}$ & 1907.19 & 2210.03 & $\mathbf{5 7 1 8 . 3 3}$ \\
\hline VI & 1423.49 & 1137.28 & 1252.06 & 2373.62 & 1907.19 & $\mathbf{0}$ & 227.75 & $\mathbf{4 9 5 3 . 0 3}$ \\
\hline VII & 1823.34 & 1373.49 & 1644.16 & 2781.26 & 2210.03 & 227.75 & $\mathbf{0}$ & $\mathbf{5 1 7 7 . 8 3}$ \\
\hline VIII & $\mathbf{4 3 0 6 . 5 2}$ & $\mathbf{2 8 2 6 . 0 1}$ & $\mathbf{7 8 2 8 . 6 6}$ & $\mathbf{1 9 3 8 . 5 5}$ & $\mathbf{5 7 1 8 . 3 3}$ & $\mathbf{4 9 5 3 . 0 3}$ & $\mathbf{5 1 7 7 . 8 3}$ & $\mathbf{6 0 8 . 1 4}$ \\
\hline
\end{tabular}

Table.5 Cluster mean values for different characters of finger millet genotypes under moisture stress free environment

\begin{tabular}{|l|l|l|l|l|l|l|l|l|l|l|l|l|l|l|l|}
\hline Cluster & $\mathbf{X}_{\mathbf{1}}$ & $\mathbf{X}_{\mathbf{2}}$ & $\mathbf{X}_{\mathbf{3}}$ & $\mathbf{X}_{\mathbf{4}}$ & $\mathbf{X}_{\mathbf{5}}$ & $\mathbf{X}_{\mathbf{6}}$ & $\mathbf{X}_{\mathbf{7}}$ & $\mathbf{X}_{\mathbf{8}}$ & $\mathbf{X}_{\mathbf{9}}$ & $\mathbf{X}_{\mathbf{1 0}}$ & $\mathbf{X}_{\mathbf{1 1}}$ & $\mathbf{X}_{\mathbf{1 2}}$ & $\mathbf{X}_{\mathbf{1 3}}$ & $\mathbf{X}_{\mathbf{1 4}}$ & $\mathbf{X}_{\mathbf{1 5}}$ \\
\hline I & 77.3 & 109.8 & 96.1 & 3.0 & 7.3 & 7.1 & 3.3 & 30.0 & 31.3 & 26.4 & 7.0 & 8.8 & 84.3 & 32.3 & 25.4 \\
\hline II & 61.4 & 92.7 & 77.3 & 3.2 & 7.5 & 6.9 & 3.2 & 31.0 & 27.3 & 23.5 & 7.0 & 8.6 & 82.7 & 35.2 & 23.9 \\
\hline III & 71.0 & 102.20 & 113.0 & 3.2 & 6.3 & 7.5 & 3.2 & 20.3 & 36.3 & 30.3 & 8.2 & 5.3 & 82.6 & 28.6 & 17.1 \\
\hline IV & 77.6 & 111.65 & 120.7 & 3.3 & 7.3 & 7.4 & 3.4 & 31.8 & 30.5 & 26.3 & 7.0 & 8.9 & 84.3 & 35.0 & 26.3 \\
\hline V & 91.0 & 124.7 & 97.1 & 2.4 & 7.2 & 6.8 & 3.3 & 36.1 & 33.2 & 25.9 & 6.9 & 14.0 & 89.8 & 36.8 & 24.8 \\
\hline VI & 85.0 & 125.0 & 80.0 & 4.2 & 7.2 & 7.9 & 3.9 & 25.8 & 23.6 & 21.9 & 6.5 & 9.1 & 85.6 & 39.6 & 20.5 \\
\hline VII & 78.0 & 109.0 & 63.6 & 2.8 & 8.2 & 7.2 & 3.9 & 37.1 & 27.8 & 24.0 & 6.2 & 7.9 & 80.7 & 32.6 & 34.9 \\
\hline
\end{tabular}

$\mathbf{X}_{\mathbf{1}}=$ Days to $50 \%$ flowering $\quad \mathbf{X}_{\mathbf{5}}=$ Finger length $(\mathrm{cm}) \mathbf{X}_{\mathbf{9}}=$ Root length $(\mathrm{cm}) \mathbf{X}_{\mathbf{1 3}}=\mathrm{RWC}(\%)$

$\mathbf{X}_{\mathbf{2}}=$ Days to maturity $\quad \mathbf{X}_{\mathbf{6}}=$ Fingers/earhead $\quad \mathbf{X}_{\mathbf{1 0}}=$ Root volume (cc) $\mathbf{X}_{\mathbf{1 4}}=$ SCMR reading

$\mathbf{X}_{\mathbf{3}}=$ Plant height $(\mathrm{cm}) \quad \mathbf{X}_{\mathbf{7}}=1000$ Seed weight $(\mathrm{g}) \mathbf{X}_{\mathbf{1 1}}=$ Root biomass $(\mathrm{g}) \mathbf{X}_{15}=$ Grain yield $(\mathrm{g} / \mathrm{plant})$

$\mathbf{X}_{\mathbf{4}}=$ Productive tillers/plant $\mathbf{X}_{\mathbf{8}}=$ Harvest index $(\%) \mathbf{X}_{\mathbf{1 2}}=$ Proline $\left(\mu\right.$ mole $\mathrm{g}^{-1}$ fr.wt $)$

Table.6 Cluster mean values for different characters of finger millet genotypes under terminal moisture stress environment

\begin{tabular}{|c|c|c|c|c|c|c|c|c|c|c|c|c|c|c|c|}
\hline Clusters & $\mathbf{X}_{\mathbf{1}}$ & $\mathbf{X}_{\mathbf{2}}$ & $\mathbf{X}_{\mathbf{3}}$ & $\mathbf{X}_{\mathbf{4}}$ & $\mathbf{X}_{\mathbf{5}}$ & $\mathbf{X}_{\mathbf{6}}$ & $\mathbf{X}_{\mathbf{7}}$ & $\mathbf{X}_{\mathbf{8}}$ & $\mathbf{X}_{\mathbf{9}}$ & $\mathbf{X}_{\mathbf{1 0}}$ & $\mathbf{X}_{\mathbf{1 1}}$ & $\mathbf{X}_{\mathbf{1 2}}$ & $\mathbf{X}_{\mathbf{1 3}}$ & $\mathbf{X}_{\mathbf{1 4}}$ & $\mathbf{X}_{\mathbf{1 5}}$ \\
\hline $\mathbf{I}$ & 75.6 & 107.8 & 88.3 & 2.0 & 6.5 & 6.4 & 2.8 & 28.3 & 33.7 & 25.0 & 7.13 & 15.6 & 72.1 & 28.7 & 21.4 \\
\hline II & 70.7 & 107.4 & 104.0 & 2.0 & 7.3 & 7.0 & 2.6 & 22.5 & 33.5 & 25.1 & 7.0 & 13.1 & 63.9 & 24.5 & 16.1 \\
\hline III & 57.3 & 91.2 & 71.2 & 2.3 & 7.3 & 6.7 & 2.8 & 28.3 & 31.3 & 23.7 & 7.1 & 15.5 & 73.2 & 30.0 & 20.7 \\
\hline IV & 74.5 & 108.3 & 116.7 & 2.2 & 6.7 & 7.0 & 2.8 & 29.7 & 33.7 & 26.1 & 7.1 & 16.2 & 75.4 & 31.3 & 21.1 \\
\hline V & 79.7 & 112.7 & 78.4 & 2.0 & 7.4 & 7.4 & 2.8 & 23.8 & 27.5 & 24.3 & 6.8 & 12.6 & 62.0 & 24.7 & 17.5 \\
\hline VI & 56.0 & 90.0 & 91.5 & 1.2 & 5.8 & 6.5 & 2.2 & 17.0 & 27.1 & 21.5 & 7.3 & 13.8 & 60.3 & 20.4 & 14.3 \\
\hline VII & 56.5 & 88.6 & 92.4 & 2.4 & 12.0 & 8.1 & 2.0 & 21.9 & 31.9 & 14.2 & 6.8 & 10.1 & 52.4 & 19.5 & 11.8 \\
\hline VIII & 82.5 & 114.0 & 148.7 & 2.1 & 7.23 & 7.5 & 2.7 & 28.1 & 24.2 & 23.0 & 7.1 & 13.2 & 64.5 & 26.0 & 22.4 \\
\hline
\end{tabular}

$\mathbf{X}_{\mathbf{1}}=$ Days to $50 \%$ flowering $\quad \mathbf{X}_{\mathbf{5}}=$ Finger length $(\mathrm{cm}) \mathbf{X}_{\mathbf{9}}=$ Root length $(\mathrm{cm}) \mathbf{X}_{13}=$ RWC (\%)

$\mathbf{X}_{2}=$ Days to maturity $\quad \mathbf{X}_{\mathbf{6}}=$ Fingers/earhead $\quad \mathbf{X}_{10}=$ Root volume (cc) $\mathbf{X}_{14}=$ SCMR reading

$\mathbf{X}_{\mathbf{3}}=$ Plant height $(\mathrm{cm}) \quad \mathbf{X}_{\mathbf{7}}=1000$ Seed weight $(\mathrm{g}) \mathbf{X}_{\mathbf{1 1}}=$ Root biomass $(\mathrm{g}) \mathbf{X}_{\mathbf{1 5}}=$ Grain yield $(\mathrm{g} / \mathrm{plant})$

$\mathbf{X}_{\mathbf{4}}=$ Productive tillers/plant $\mathbf{X}_{\mathbf{8}}=\operatorname{Harvest}$ index $(\%) \mathbf{X}_{\mathbf{1 2}}=$ Proline $\left(\mu\right.$ mole g $\mathrm{g}^{-1}$ fr.wt $)$ 
Table.7 Contribution of different characters towards genetic divergence in finger millet under moisture stress free environment

\begin{tabular}{|c|c|c|c|}
\hline Sl. No. & Characters & No. of first rank & Contribution $\mathbf{~}$ \\
\hline $\mathbf{1}$ & Days to 50\% flowering & 829 & 11.07 \\
\hline $\mathbf{2}$ & Days to maturity & 72 & 1.18 \\
\hline $\mathbf{3}$ & Plant height $(\mathrm{cm})$ & 537 & 10.06 \\
\hline $\mathbf{4}$ & Productive tillers/plant & 0 & 0 \\
\hline $\mathbf{5}$ & Finger length $(\mathrm{cm})$ & 7 & 0.11 \\
\hline $\mathbf{6}$ & Fingers/earhead & 18 & 0.75 \\
\hline $\mathbf{7}$ & 1000 seed weight $(\mathrm{g})$ & 201 & 3.29 \\
\hline $\mathbf{8}$ & Harvest index $(\%)$ & 97 & 1.59 \\
\hline $\mathbf{9}$ & Root length $(\mathrm{cm})$ & 72 & 1.18 \\
\hline $\mathbf{1 0}$ & Root volume $(\mathrm{cc})$ & 49 & 0.8 \\
\hline $\mathbf{1 1}$ & Root biomass $(\mathrm{g})$ & 0 & 0 \\
\hline $\mathbf{1 2}$ & Proline $\left(\mu\right.$ mole $\mathrm{g}^{-1}$ fr.wt) & 6 & 0.1 \\
\hline $\mathbf{1 3}$ & RWC $(\%)$ & 248 & 3.46 \\
\hline $\mathbf{1 4}$ & SCMR & 916 & 15.64 \\
\hline $\mathbf{1 5}$ & Grain yield $\left(\mathrm{g}\right.$ plant $\left.{ }^{-1}\right)$ & 3053 & 50.77 \\
\hline
\end{tabular}

Table.8 Contribution of different characters towards genetic divergence in finger millet under terminal moisture stress environment

\begin{tabular}{|c|c|c|c|}
\hline Sl. No. & Characters & No. of first rank & Contribution \% \\
\hline $\mathbf{1}$ & Days to 50\% flowering & 819 & 11.07 \\
\hline $\mathbf{2}$ & Days to maturity & 646 & 10.74 \\
\hline $\mathbf{3}$ & Plant height $(\mathrm{cm})$ & 343 & 7.79 \\
\hline $\mathbf{4}$ & Productive tillers/plant & 0 & 0 \\
\hline $\mathbf{5}$ & Finger length $(\mathrm{cm})$ & 2 & 0.03 \\
\hline $\mathbf{6}$ & Fingers/earhead & 7 & 0.11 \\
\hline $\mathbf{7}$ & 1000 seed weight $(\mathrm{g})$ & 241 & 3.95 \\
\hline $\mathbf{8}$ & Harvest index $(\%)$ & 154 & 2.59 \\
\hline $\mathbf{9}$ & Root length $(\mathrm{cm})$ & 103 & 1.59 \\
\hline $\mathbf{1 0}$ & Root volume $(\mathrm{cc})$ & 23 & 0.38 \\
\hline $\mathbf{1 1}$ & Root biomass $(\mathrm{g})$ & 0 & 0 \\
\hline $\mathbf{1 2}$ & Proline $\left(\mu \mathrm{mole} \mathrm{g}^{-1}\right.$ fr.wt) & 5 & 0.08 \\
\hline $\mathbf{1 3}$ & RWC $(\%)$ & 713 & 10.81 \\
\hline $\mathbf{1 4}$ & SCMR & 14 & 0.23 \\
\hline $\mathbf{1 5}$ & Grain yield $\left(\mathrm{g}\right.$ plant $\left.{ }^{-1}\right)$ & 3035 & 50.63 \\
\hline
\end{tabular}

Under terminal moisture stress (TMS), as far as cluster means are concerned, cluster I had the highest mean value for root length and the lowest mean for fingers per ear head. Cluster III had lowest mean for plant height. Cluster
IV had the highest mean values for harvest index, root volume, proline, RWC and SCMR. Cluster $\mathrm{V}$ had the highest mean value for 1000 seed weight. Cluster VI had the highest mean value for root biomass. Cluster 
VII had highest mean values for productive tillers per plant, finger length and fingers per ear head. Cluster VIII had highest mean values for days to fifty per cent flowering, days to maturity, plant height and grain yield, while it showed lowest mean for root length. Equivalent results have also been reported by Anteneh et al., (2019), Kandel et al., (2019) and Swamynatham et al., (2020).

The relative contribution of different characters towards genetic divergence under MSF and TMS are depicted in Table 7 and Table 8. Out of the total contribution of different characters towards genetic divergence under non-stress environment, grain yield $(50.77 \%)$ showed maximum contribution towards genetic diversity. The other characters viz., SCMR (49.23\%), days to $50 \%$ flowering $(11.07 \%)$, plant height (10.06\%), RWC (3.46\%), 1000 seed weight $(3.29 \%)$, harvest index $(1.59 \%)$, days to maturity $(1.18 \%)$, root length $(1.18 \%)$, root volume $(0.8 \%)$, fingers per ear $(0.75 \%)$, finger length $(0.11 \%)$ and proline $(0.1 \%)$ contributed towards genetic divergence under MSF environment. This indicates the presence of variability for these traits among the studied genotypes. Analogous results were reported by Saundarya Kumari and Singh (2015) for grain yield per plant.

While under terminal moisture stress (TMS), grain yield (50.63\%) showed maximum contribution towards genetic diversity. The other characters viz., days to $50 \%$ flowering (11.07\%), RWC (10.81\%), days to maturity (10.74\%), plant height (7.79\%), 1000 seed weight $(3.95 \%)$, harvest index $(2.59 \%)$, root length $(1.59 \%)$, root volume $(0.38 \%)$, SCMR $(0.23 \%)$, fingers per ear $(0.11 \%)$, proline $(0.08 \%)$ and finger length $(0.03 \%)$ contributed towards genetic divergence under TMS environment. Therefore, these characters should be given due importance. Inter-cluster distances indicated that the accessions included in the diverse clusters would be used in further crop improvement programme. Subramanya and Ravikumar (2020) reported collaborative result for grain yield per plant and days to fifty per cent flowering.

\section{References}

Anteneh, D., Mekbib, F., Tadesse, T. and Dessalegn, Y. 2019. Genetic diversity among lowland finger millet (Eleusine coracana (L) Gaertn) accessions. Ethiop. J. Agric Sci., 29(2): 93-108.

Devaliya, S. D., Singh, M. and Vista, M. L. 2017. Character association and path analysis in finger millet (Eleusine coracana L.). Biosci. Trends., 10(31): 6690-6694.

Devi, P. B., Vijayabharathi, R., Sathyabama, S., Malleshi, N. G. and Priyadarisini, V. B. 2014. Health benefits of finger millet (Eleusine coracana L.) polyphenols and dietary fiber: a review. Int. J.Food SciTech., 51(6): 1021-1040.

Dinesh, K., Vikrant, T., Ramesh, B. B. and Sukram, P. 2010. Genetic diversity in finger millet (Eleusine coracana L.). Crop Improv., 37(1): 25-28.

Kandel, M., Dhami, N. B. and Shrestha, J. 2019. Phenotypic diversity of finger millet (Eleusine coracana (L.) Gaertn.) genotypes. Malays. J. Sustain. Agric., 3(2): 20-26.

Krishnamurthy, L., Johansen, C. and S. C. Sethi. 1999. Investigation of factors determining genotypic differences in seed yield of non-irrigated and irrigated chickpeas using a physiological model of yield determination. J. Agron. Crop Sci., 183: 9-17.

Ludlow, M. M. and Muchow, R. C. 1990. A critical evaluation of traits for improving crop yields in water limited environments. Adv Agron., 43: 07-153.

Patil, H. E., Patel, B. K., Vavdiya, P. and Pali, 
V. 2018. Breeding for quality improvement in small millets: A review. Int. J. Genet. Mol. Biol., 4(4): 148-151.

Rao, C.R. 1952. Advance statistical methods in biometric research. Wiley and Sons, New York. Pp. 390.

Rawat, L., Prasad, S., Bisht, T. S., Naithani, D. C. and Kumar, J. 2018. Assessment of genetic variability and divergence in finger millet accessions at mid hills of Uttarakhand., Int. J. Curr. Microbial. Appl. Sci. 7(11): 2912-2922.

Saundaryakumari. And Singh, S. K. 2015. Assessment of genetic diversity in promising finger millet (Eleusine coracana L.) genotypes., Int. $J$. Environ. Sci., 10(2): 825-830.

Subramanya, A. S. and Ravikumar, R.L. 2020. Genetic divergence studies in cultivated tetraploid finger millet
(Eleusine coracana L.) genotypes using $\mathrm{D}^{2}$ analysis. Int. J. Curr. Microbial. Appl. Sci., 9(1): 109-118.

Suryanarayana, L., Sekhar, D. and Rao, V. D. 2014. Genetic variability and divergence studies in finger millet (Eleusine coracana L.). Int. J. Curr. Microbial. Appl. Sci., 3(4): 931-936.

Swamynatham, S., Kumar, M. H., Reddy, D. M. and Latha, P. 2020. Genetic divergence studies in pearl millet (Pennisetum glaucum (L). R. Br). Electron. J. Plant Breed., 11(1): 76-80.

Wolie, A., Belete, K. and Dessalegn, T. 2013. Heritability, variance components and genetic advance of some yield and yield related traits in Ethiopian collections of finger millet (Eleusine coracana L.) genotypes. Afri. J. Biotechnol., 12(36): 5529-5534.

\section{How to cite this article:}

Manjunatha, V.T., L.N. Yogeesh, J.P. Nidagundi, G. Girish and Suma, T.C. 2020. Genetic Diversity Studies in Finger Millet under Terminal Moisture Stress. Int.J.Curr.Microbiol.App.Sci. 9(12): 951-959. doi: https://doi.org/10.20546/ijcmas.2020.912.115 